\section{Exogene allergische Alveolitis bei einem Dudelsackspieler - ein Fallbericht}

King J et al. Bagpipe lung; a new type of interstitial lung disease?. Thorax 2016; DOI 10.1136/ thoraxjnl-2016-208751

Wiederholtes Einatmen von Mikroorganismen, tierischen Proteinen oder chemischen Substanzen kann eine exogene allergische Alveolitis (EAA, synonym: Hypersensitivitätspneumonitis) auslösen. Die EAA kann mitunter einen tödlichen Verlauf haben. Dass die Allergene auch in Blasinstrumenten vorhanden sein können, zeigt der Fallbericht britischer Ärzte.

Bei einem 61-jährigen Mann wurde 2009, basierend auf HR-CT- und Biopsiebefunden, eine EAA diagnostiziert. Kontakt zu Vögeln oder Schimmelpilzen im Haus sowie eine Kollagenose konnten ausgeschlossen werden. Die Symptome (trockener Husten, Atemnot) verschlechterten sich stetig bis auf einen 3 monatigen Auslandsaufenthalt im Jahr 2011. Hier hatte der passionierte Dudelsackspieler sein Instrument nicht dabei. Es kam zu deutlichen Symptomverbesserungen mit der Möglichkeit, 10 km ununterbrochen zu gehen. Zuhause ging er seinem Hobby wieder nach und es setzte eine rasche Progression ein. Der Patient erhielt intermittierend $0,5 \mathrm{mg} / \mathrm{kg}$ Prednisolon.

Im April 2014 folgte die Überweisung an ein Zentrum für interstitielle Lungenerkrankungen (ILD). Die Gehstrecke war auf 20 m reduziert. Neue HR-CT-Aufnahmen zeigten zunehmende Milchglastrübungen und Mosaikmuster, die forcierte Vitalkapazität (FVC) betrug 1,48 (34\%) und die Diffusionskapazität (DLCO) 3,21 (33\%). 2009 wurde eine FVC von 2,29 (56\%) und eine DLCO von 4,89 (50\%) gemessen. Anhand der Beurteilung im ILD-Zentrum erhielt der Patient $2 \mathrm{mg} / \mathrm{kg}$ Azathioprin plus Prednisolon (Erhaltungsdosis). Das Dudelsackspielen setzte er fort.
Im September 2014 folgte eine Krankenhauseinweisung aufgrund über 6 Wochen zunehmender Atemnot und niedriger Sauerstoffsättigung. Der Patient war afebril, hypoxisch und tachypnoeisch mit mäßig erhöhten Entzündungsmarkern. Das Röntgen-Thorax ergab beidseitige interstitielle Veränderungen und einen undeutlichen linken Herzrand, das CT lieferte keinen Hinweis auf Lungenembolie und im Sputum zeigte sich eine normale Flora der oberen Atemwege. Für eine Bronchoskopie war der Patient zu schwach. Die Therapie bestand nun in der Gabe von Amoxicillin/Clavulansäure, Clarithromycin und Cotrimoxazol. Wegen des Verdachts auf eine Pilzpneumonie wurde Posaconazol hinzugenommen. Aus dem Dudelsack entnommene Proben beinhalteten 7 verschiedene Pilzarten. Der Patient starb im Oktober 2014 aufgrund einer ILD-Exazerbation.

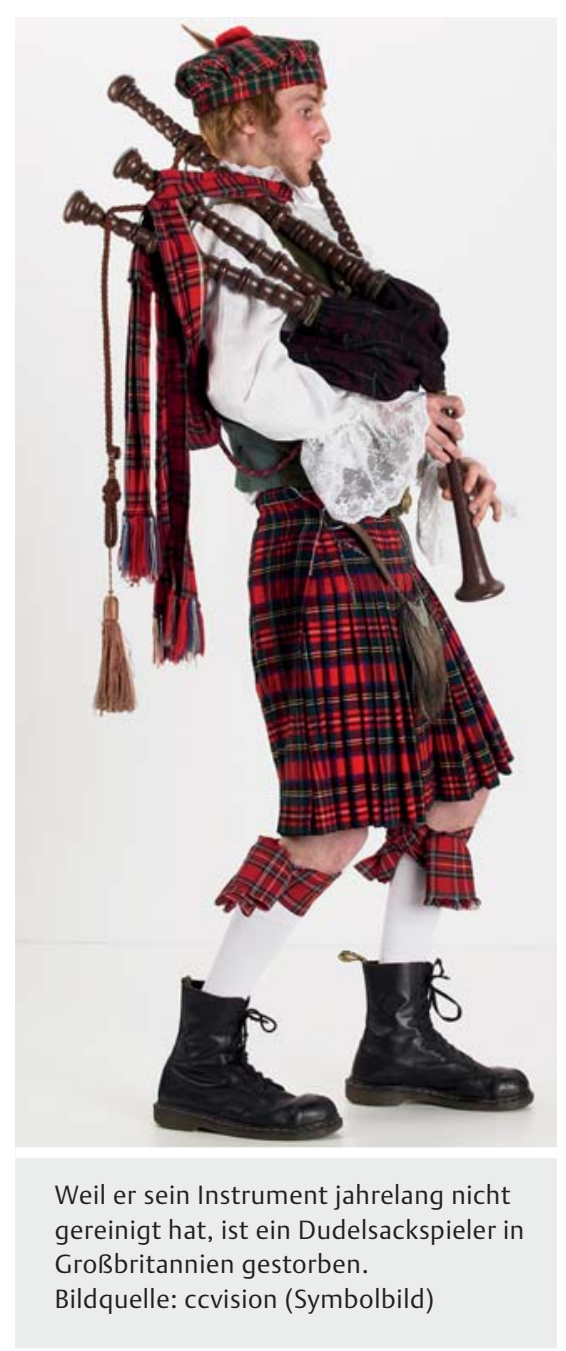

\section{FAZIT}

Dieser Bericht über eine von den Autoren sogenannte „bagpipe lung“ zeigt, dass die Pilzexposition aus einem Blasinstrument ein möglicher EAA-Auslöser sein kann. Nach ihrer Ansicht verdeutlicht dieser Fall, Hobbys wie das Spielen von Blasinstrumenten als Risikofaktor zu beachten.

Matthias Manych, Berlin 